\title{
Structure of Chinese City Network as Driven by Technological Knowl- edge Flows
}

\author{
MA Haitao ${ }^{1}$, FANG Chuanglin ${ }^{1}$, PANG Bo ${ }^{1,2}$, WANG Shaojian ${ }^{1,2}$ \\ (1. Institute of Geographic Sciences and Natural Resources Research, Chinese Academy of Sciences, Beijing 100101, China; 2. Univer- \\ sity of Chinese Academy of Sciences, Beijing 100049, China)
}

\begin{abstract}
Based on patent cooperation data, this study used a range of city network analysis approaches in order to explore the structure of the Chinese city network which is driven by technological knowledge flows. The results revealed the spatial structure, composition structure, hierarchical structure, group structure, and control structure of Chinese city network, as well as its dynamic factors. The major findings are: 1) the spatial pattern presents a diamond structure, in which Wuhan is the central city; 2) although the invention patent knowledge network is the main part of the broader inter-city innovative cooperation network, it is weaker than the utility model patent; 3 ) as the senior level cities, Beijing, Shanghai and the cities in the Zhujiang (Pearl) River Delta Region show a strong capability of both spreading and controlling technological knowledge; 4) whilst a national technology alliance has preliminarily formed, regional alliances have not been adequately established; 5) even though the cooperation level amongst weak connection cities is not high, such cities still play an important role in the network as a result of their location within 'structural holes' in the network; and 6) the major driving forces facilitating inter-city technological cooperation are geographical proximity, hierarchical proximity and technological proximity.
\end{abstract}

Keywords: technological knowledge flows; patent cooperation; city networks; network structure; structure holes; cohesive subgroup

Citation: Ma Haitao, Fang Chuanglin, Pang Bo, Wang Shaojian, 2015. Structure of Chinese city network as driven by technological knowledge flows. Chinese Geographical Science, 25(4): 498-510. doi: 10.1007/s11769-014-0731-0

\section{Introduction}

City networks, read as a product of the transition from a 'space of places' to 'space of flows' (Bourne, 1975; Castells, 1989; Neal, 2010), is a hot topic in the field of urban geography (Esparza and Krmenec, 2000), and have been since the turn of this century (Camagni and Capello, 2004). The form and structure of city networks are understood as basing on a number of different 'flows', which are distinct from each other. The importance of each type of flow in each city network in turn changes over time. A number of scholars have carried out research into city networks based on various kinds of flow (Taylor, 2001; Taylor et al, 2002; Derudder and Witlox, 2005; Choi et al., 2006; Dai and Jin, 2008; Mo et al., 2009; Jacobs et al., 2010; Neal, 2010; Vinciguerra et al., 2010; Leng et al., 2011; Wang et al., 2011). Whilst all of these studies have greatly enriched the city networks research and promoted the development of an academic city networks theory, little attention has been paid to knowledge flows in the context of the knowledge economy (Lu and Huang, 2012).

Against the background of the emergence of the knowledge economy, the Chinese government has proposed the grand goal of building an innovation-oriented nation by 2020. As important platforms for innovation, widespread attention has been paid to the construction of innovative cities, and as a result inter-city collaborative innovation and knowledge communication have been strengthened. Despite these improvements, how-

Received date: 2014-04-28; accepted date: 2014-08-11

Foundation item: Under the auspices of Major Project of National Social Science Foundation of China (No. 13\&ZD027), National Natural Science Foundation of China (No. 41201128, 71433008)

Corresponding author: FANG Chuanglin. E-mail: fangcl@igsnrr.ac.cn

(C) Science Press and Northeast Institute of Geography and Agroecology, CAS and Springer-Verlag Berlin Heidelberg 2015 
ever, most urban innovation studies have focused on evaluating cities' innovative capabilities and their inner spaces of innovation, neglecting to address both innovative collaboration between cities, and the broader urban knowledge network (Lu and Huang, 2012). Research methods from the field of city networks can help scholars to explore knowledge flows, knowledge transfer, knowledge spillover and knowledge aggregation at the national level, and to grasp the internal structure of cooperative knowledge innovation. In turn, the results of such research can also provide a reference for the central government and local governments in forming general opinions on innovation patterns, as well as formulating innovation policies and planning programs.

The co-production of knowledge has become a central issue in recent years, and research into such co-production not only benefits the construction of scientific alliances, but can also help policy makers (city governments) understand the role of geographical space in research cooperation more profoundly (Nascimbeni, 2013). Propelled by bibliometric methods and associated techniques, the measure of cooperative academic papers has therefore received unprecedented attention as a basis for understanding the co-production of knowledge, and of knowledge networks. Most studies using this measure have either taken researchers (Hou et al., 2008) or research institutes (Leydesdorff and Persson, 2010) - rather than cities - as nodes in their analysis of a given knowledge network. As a result, such studies have tended to overplay the role of 'intra-city' cooperation (that is, cooperation between a high number of researchers or research institutes within the same city). Generally speaking, intra-city cooperation in fact reduces the innovative vitality of a broader knowledge network by producing redundant knowledge, because within just one city there is a consistent scientific environment and the same local knowledge pool. Alternately, since each city has its own scientific resources and technological expertise, complementary cooperation between cities (that is, 'inter-city' cooperation) would be of far greater assistance in improving knowledge diversity and establishing a more complete research chain. As such, knowledge network research using city-based nodes (rather than researchers or institutions) can generate more valuable findings; for instance, revealing more about the status of a particular city in knowledge transfer and spread (Matthiessen et al., 2010), the key central scientific cities in a broader system (Matthiessen et al., 2002), or the spatial form of a particular knowledge network (Liefner and Hennemann, 2011).

Knowledge can be divided into scientific knowledge and technological knowledge. Practices must be developed in order to transform scientific knowledge into technological knowledge, as the latter seems more effective than the former in achieving rapid improvements in a country's innovation capability. Like the measure of cooperatively produced papers, patent cooperation is also used to examine the technological knowledge network. For example, in this vein scholars have investigated the footprint of technological knowledge spinoff (Thompson and Fox-Kean, 2005; Fischer et al., 2006; Tang and $\mathrm{Hu}, 2013)$, technological collaboration patterns (Lei et al., 2013), technological communities (Barber and Scherngell, 2013), and the impact of technological cooperation on innovation (Eslami et al., 2013). Despite these examples, however, explorations of technological knowledge networks remain insufficient, and it is this lack which lays the foundation and provides the opportunity for this study to contribute to the field.

Based on the above considerations, the study detailed in this paper used patent cooperation data and various city network analysis approaches to explore the Chinese city network structure.

\section{Materials and Methods}

\subsection{Data}

Patent analysis is considered to be particularly appropriate for probing geographical collaboration in inventive activities. Patent data represent a valuable source of information in relation to technological development and collaboration (Eslami et al., 2013), and several of the information fields included in a patent application can yield valuable insights into inventive activities. Inventors usually provide their detailed address information when applying for a patent, which makes patent applications suitable for the analysis of technological collaborations within geographical space (Lei et al., 2013). Given that patent collaboration can be divided into three types, that is, local collaboration, domestic collaboration, and international collaboration, this article focuses on domestic collaboration, through the analysis of patent applications from inventors who have addresses in dif- 
ferent cities in the same country (in this case, China).

Using data from the Chinese Patent Database of the China National Knowledge Infrastructure (CNKI) platform, this study explored the annual number of cooperative patent applications submitted in 2012, wherein cooperation occurred between 60 selected cities (Beijing, Tianjin, Shijiazhuang, Tangshan, Qinhuangdao, Jinan, Qingdao, Yantai, Shanghai, Nanjing, Wuxi, Zhenjiang, Changzhou, Suzhou, Xuzhou, Lianyungang, Hangzhou, Ningbo, Jiaxing, Wenzhou, Fuzhou, Xiamen, Quanzhou, Guangzhou, Shenzhen, Dongguan, Foshan, Zhongshan, Zhuhai, Haikou, Taiyuan, Zhengzhou, Luoyang, Wuhan, Jingzhou, Changsha, Nanchang, Hefei, Harbin, Daqing, Changchun, Jilin, Shenyang, Dalian, Hohhot, Baotou, Yinchuan, Xi'an, Baoji, Lanzhou, Xining, Chengdu, Chongqing, Guiyang, Nanning, Liuzhou, Guilin, Kunming, Ürümqi, Lhasa). We constructed a $60 \times 60$ inter-city patent cooperation matrix, with multi-weights and symmetry features, which was able to demonstrate technological links between cities. When selecting cities to include in this matrix, two factors were considered: first, a city's capability in terms of urban technological connections, whereby cities with the greatest patent cooperation were chosen; second, regional balancing, whereby at least one city was selected in each province, and all the provincial capital cities were selected.

\subsection{Methods}

This study drew upon the approaches of spatial network analysis, social network analysis and network evolution analysis in order to explore Chinese technological cooperation network structure. Because the inter-city technological cooperation network discussed here is based on the technological knowledge flows between patent inventors, when using social network analysis, we regarded the city actors to be human actors. Indicators used for measurement, as well as the calculation methods used, are detailed below.

\subsubsection{Network connectivity degree}

The Intercity Network Connectivity Degree $\left(R_{a b}\right)$ suggests the degree of technological cooperation between city $a$ and city $b$. Here, it can be represented by the actual number of instances of patent cooperation between city $a$ and city $b\left(V_{a b}\right)$.

$R_{a b}=V_{a b}$

The City Total Network Connectivity Degree $\left(N_{a}\right)$ in- dicates the technological cooperation capability of a single city $a$, which can be expressed by the sum of all the connectivity degrees between one city and the others. The formula is below:

$$
N_{a}=\sum_{i=1}^{n} R_{a i}
$$

where $R_{a i}$ is the intercity network connectivity degree between city $a$ and city $i$.

\subsubsection{Network density}

Network Density $(D)$, calculated as the actual number of technological connections divided by the theoretically possible number of technological connections, was used to measure network health and effectiveness. The higher the density is, the closer the inter-city technological connections are. $D$ is given by the equation:

$D=2 m / n(n-1)$

where $n$ is the number of cities, $m$ is the actual number of connections in the network.

\subsubsection{Network centrality}

Network Centrality refers to a city's capability to dominate technological knowledge in a technological cooperation network, through measures of Local Centrality, Betweenness Centrality and Closeness Centrality.

Local Centrality measures the ability of a city to carry out technological cooperation with other cities, using the following formula:

$$
C_{a d}=N_{a}=\sum_{i=1}^{n} R_{a i}
$$

where $C_{a d}$ is the local centrality of city $a ; N_{a}$ is the technological cooperation capability of a single city $a$; and $R_{a i}$ is the intercity network connectivity degree between city $a$ and city $i$.

Betweenness Centrality was used to measure the controlling degree of a city on technological knowledge. Its expression is as follows:

$$
C_{a b}=\sum_{j}^{n} \sum_{k}^{n} G_{j k}(a) / G_{j k}
$$

where $C_{a b}$ is the betweenness centrality of city $a ; G_{j k}$ indicates the number of geodesic path between city $j$ and city $k ; G_{j k}(a)$ describes the number of geodesic paths between city $j$ and city $k$, which pass city $a$. The geodesic path is the strongest connective path between two cities. 
Closeness Centrality measures the degree of freedom a city has in terms of not being controlled by other cities. Its expression is as follows:

$C_{a p}=1 / \sum_{i=1}^{n} G_{a i}$

where $C_{a p}$ is the closeness centrality of city $a$; $\sum_{\mathrm{i}=1}^{n} G_{a i}$ represents the sum of geodesic paths between city $a$ and all other cities.

\subsubsection{Structural hole indicator}

Effective Size (ES) was used to measure a city's capability to control technological knowledge spread in a technological cooperation network. The more effective the city's capability in controlling technological knowledge, the higher the ES value is. Higher ES values also reflect greater non-redundant knowledge and stronger technological competitiveness in a given city. Its expression is as follows:

$E S=\sum_{j}\left(1-\sum_{q} p_{a q} m_{j q}\right), q \neq a, j$

where $j$ represents all the cities connected to the ego city; $q$ is the individual third party except $a$ and $j$; and $p_{a q} m_{j q}$ indicate the redundancy between ego city and $j$.

Efficiency $(E f)$, calculated by the city's effective scale divided by its actual scale, measures the city's degree of control over the technological knowledge spread in the technological cooperation network. When $E f$ equals 1, the effective scale is the same as the actual scale. Its expression is as follows:

$E f=E S / n=\sum_{j}\left(1-\sum_{q} p_{a q} m_{j q}\right) / n, q \neq a, j$

where $n$ is the actual individual network scale of ego city $a$, other variables are the same as those in Equation (7).

Constraint degree $\left(C_{a}\right)$ refers to a city's ability to utilize structural holes; it measures a given city's degree of direct and indirect dependence in relation to its technological knowledge spread on other cities. The higher the $C_{a}$ value is, the stronger the limitation from technological cooperation is. Its expression is as follows:

$$
C_{a}=\sum_{j}\left(p_{i j}+\sum_{q} p_{a q} m_{j q}\right)^{2}, q \neq a, j
$$

where $C_{a}$ is the constraint degree of city $a$, other variables are the same as those in Equation (7).

\section{Results}

By using patent cooperation data and various city networks analysis approaches, we undertook a comprehensive analysis of the Chinese inter-city network structure as it is driven by technological knowledge flow, revealing the spatial structure (using GIS methods), the composition structure (through patent type analysis), the hierarchical structure (through centrality analysis), the group structure (via cohesive subgroup analysis), the control structure (using Structural Holes Theory), and the dynamic factors (from the perspective of proximity) of China's inter-city technological cooperation network.

\subsection{City network spatial structure based on GIS}

Using the patent data and GIS analysis, the structure of the technological knowledge cooperation network of 60 cities could be determined; this network was found to take the form of a diamond structure (Fig. 1a). All the cities were contained in this technological knowledge cooperation network, with the sole exceptions of Lhasa, Haikou and Jingzhou. Within the network, patent cooperation occurred between 622 city pairs, with the total network connectivity degree being 6738 . The average connectivity degree was found to be 10.83 , and the general network density to be 1.90 . From the point of view of spatial structure, five prominent cities or urban areas emerged: namely, Beijing, Wuhan, urban areas in the Changjiang (Yangtze) River Delta, urban areas in the Pearl River Delta and urban areas in Chengdu-Chongqing region, whose closer relations result in the diamond structure. The supporting capability, however, was not found to be the same between each city point, the significant advantages occurred in Beijing, in urban areas in the Changjiang River Delta, and in urban areas in the Zhujiang River Delta (among which Beijing, with an network connectivity degree of 753 , had the strongest supporting ability as a single point). The total network connectivity degree of cities in the Changjiang River Delta was 2067, and the average connectivity degree was 229.67. Further, the total network connectivity degree of cities in Zhujiang River Delta was 2033, and the average connectivity degree was 338.83 . As a result, the strong connectivity between the above three urban areas 

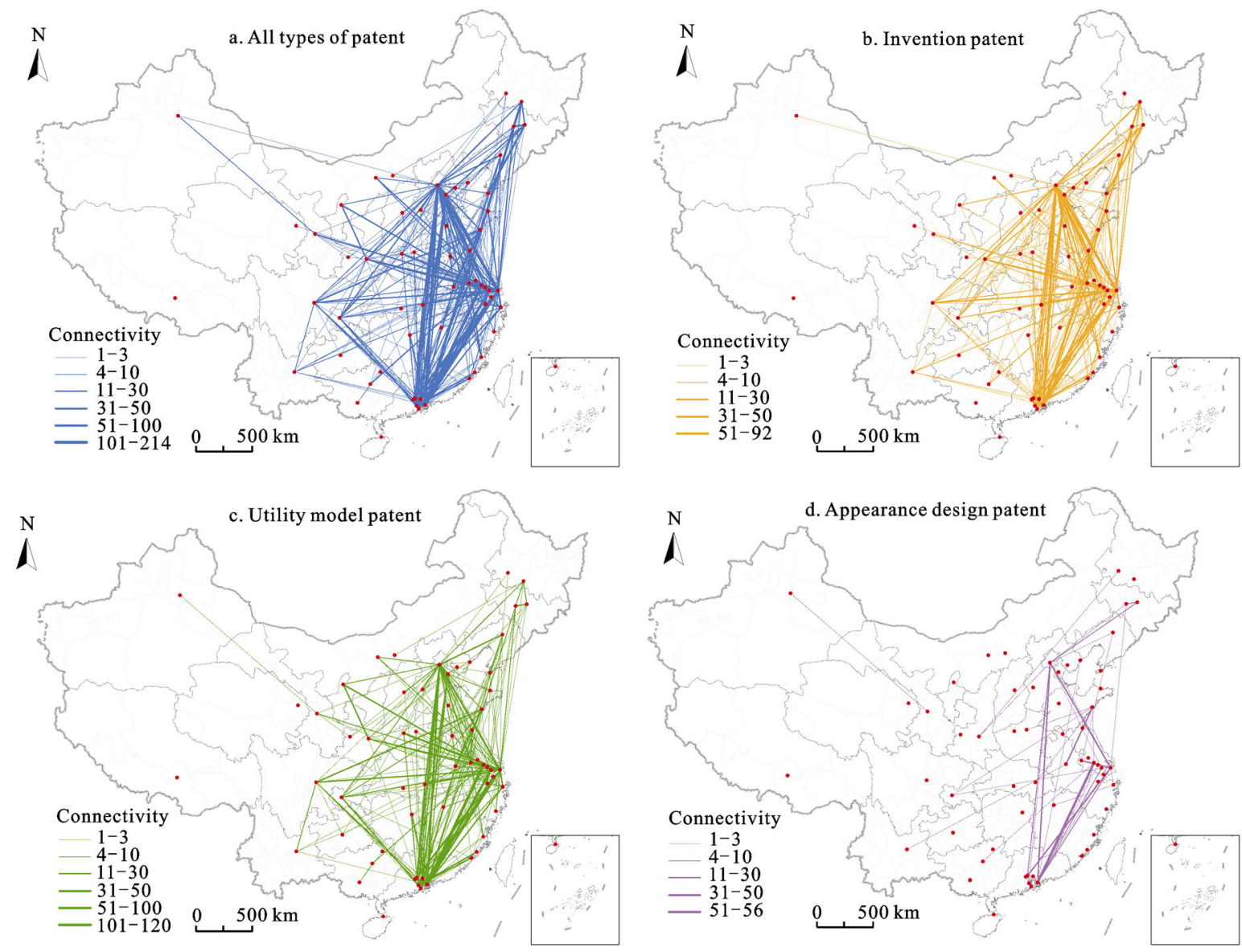

Fig. 1 Urban technological networks denoted by all type of patents (a) and three types of patents (b, c, d) in China

shows up as an obvious triangle in the diamond-like structure. Located in the center of this structure, Wuhan had a network connectivity degree of 124 , ranking 15 among the 60 cities, indicating that Wuhan is not performing very well as the mediator in connection activities among the eastern, western, southern and northern parts of China. Moreover, the external technological connectivity of Chengdu-Chongqing region was revealed as being relatively weak, indicating that the region can not provide strong support for the diamond structure similarly. Meanwhile, inter-city connections in coastal regions were found to be relatively close, forming a strong connection belt. The diamond structure generated through this study is felt to generally reflect the overall pattern of Chinese urban-technological cooperation.

\subsection{Network composition structure based on pat- ent types}

Different types of patent maintain different levels of development and produce different network forms. In China, three main types of patent exist: invention patents, utility model patents and appearance design patents. Among these, the utility model patent network is the most developed. The findings of this study indicate that this patent type has the highest values in terms of network scale, total network connectivity degree, average connectivity degree, and network density. In comparison, the development of the invention patent network was revealed as being slightly inferior, with the exception of the number of connection pairs, other indicators were found to be lower than those generated in relation to the utility model patent. In comparison, the appearance design patent network was revealed to be insufficiently developed, with just 32 cities participating in cooperation through applications for this type of patent, its total network connectivity degree accounted for just $7.66 \%$ of the whole network, and its network density was only 0.14 (Fig. 1b, Fig. 1c, Fig. 1d and Table 1). In general, the utility model patent and the invention 
Table 1 A comparative analysis of city networks driven by different types of patent knowledge

\begin{tabular}{lcccc}
\hline \multicolumn{1}{c}{ Type of network } & All types of patent & Invention patent & Utility model patent & Appearance design patent \\
\hline Network size & 57 & 56 & 56 & 32 \\
Total network connectivity degree (proportion) & $6738(100 \%)$ & $2944(43.69 \%)$ & $3278(48.65 \%)$ & $516(7.66 \%)$ \\
City pairs & 622 & 490 & 444 & 84 \\
Average connectivity degree & 10.8328 & 6.0082 & 7.3829 & 6.1429 \\
Network density & 1.9034 & 0.8316 & 0.9260 & 0.1458 \\
\hline
\end{tabular}

patent were shown to be the main supporting elements in Chinese inter-city technological cooperation, together making up for $92.34 \%$ of the total cooperation on patent applications. The amount of cooperation being performed in relation to the invention patent (which represents the top-level patent) was found to be slightly lower than in the case of the utility model patent. It is likely that the appearance design patent does not require as much inter-city cooperation as a result of the type of knowledge that it represents.

\subsection{Hierarchical structure based on centrality analysis}

Each city maintains a different degree of 'centrality', a measure which reflects its capacity to dominate technological knowledge within a given technological cooperation network. Analysis of the structure of that centrality can help us to understand patterns of urban technological knowledge aggregation. In order to measure centrality, multi-weighted networks must be transformed into binary networks. In this study, the median value of all the cities' relations (which was 3 ) was selected as the cut-off value in order to transform the relation-weighted urban network into a binary network. Because links with relation values equal to or less than 3 were neglected, the isolated points in the network increased from 4 to 11, with Zhenjiang, Haikou, Jingzhou, Daqing, Hohhot, Baoji, Xi'ning, Guiyang, Nanning, Guilin and Lhasa all being considered isolated in the new binary network. Since these isolated points can affect centrality analysis, they were excluded from the binary network, and a new relation matrix and network was constructed with the remaining 49 cities, which was then subsequently used to calculate the various centrality values for each city.

From the local centrality $\left(C_{a d}\right)$ values generated (Fig. 2a), hierarchical differences were evident in the capacity of Chinese cities to engage in inter-city technological cooperation. Findings reveal the top eight cities to be Beijing, Shanghai, Shenzhen, Nanjing, Guangzhou,
Suzhou, Tianjin and Zhongshan. An analysis of betweenness centrality $\left(C_{a b}\right)$ values (Fig. $\left.2 b\right)$ showed Beijing and Shanghai to have an absolute advantage in dominating technological knowledge. These two cities were characterized by betweenness centrality values of 37.15 and 31.91 respectively, which are much higher than that of Shenzhen (8.04), which ranked third on the list. Further, 14 cities were found to have betweenness centrality values of less than 1 , and 19 cities betweenness centrality values of zero, which indicates that most of the cities studied do not at present possess the capacity to dominate technological knowledge within the Chinese technological cooperation network. In comparison to betweenness centrality, an analysis of closeness centrality $\left(C_{a p}\right)$ (Fig. $2 \mathrm{c}$ ) reveals a lower degree of domination amongst the cities of the network. The maximum value of closeness centrality was found to be 72.73 , occurring in Beijing and Shanghai. The minimum value was 30.38 , in Ürümqi. This clearly demonstrates a smaller difference between the top and bottom of the hierarchy. The study reveals that most cities in China would likely choose Beijing and Shanghai as cooperation partners, specifically, 44 cities (or $89.79 \%$ ) were found to be cooperating with Beijing, whilst 40 cities (or $81.63 \%$ ) were cooperating with Shanghai. These high percentages constitute the main reason for a widely higher value of closeness centrality. In general, regardless of their scored in terms of local centrality, betweenness centrality and closeness centrality, Beijing and Shanghai clearly constitute two absolute centers in the Chinese technological cooperation network, ranking first and second respectively (with Shanghai's values being slightly lower than those of Beijing).

Whilst any given city's ability to carry out technological cooperation, to control technological knowledge spread and its approach to high-tech centers is usually consistent, some cities possess obvious advantages with respect to just one aspect. By conducting a Pearson 

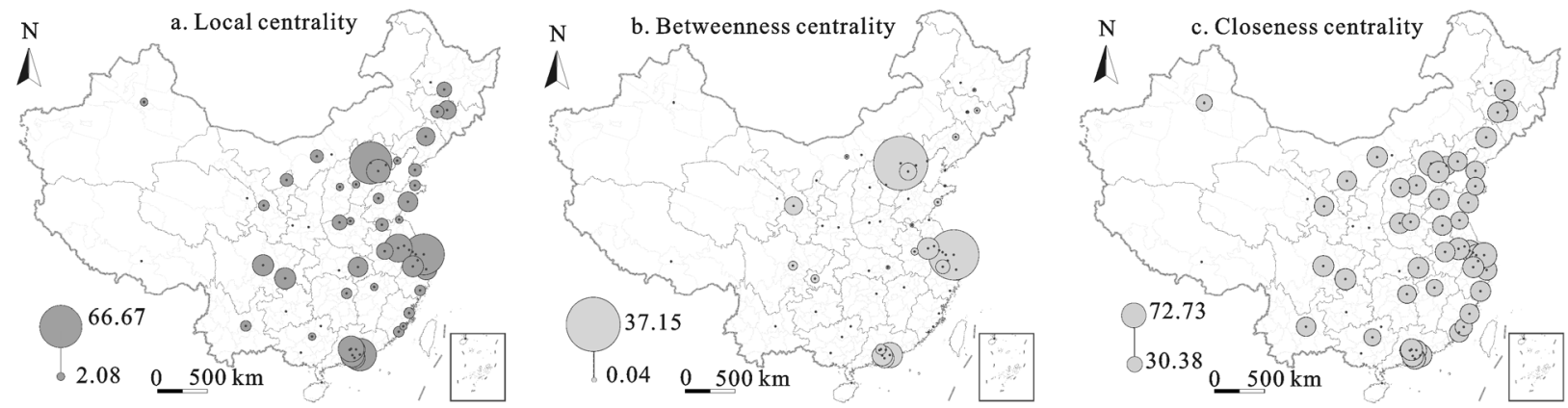

Fig. 2 Centrality analysis of 49 cities based on patent collaboration

correlation analysis on the three centrality types addressed by the study, we were able to generate regression coefficients between each centrality type and the other two types. These coefficients were $0.906,0.887$ and 0.740 . They were all significant at the 0.01 level, indicating a strong correlation between the three centralities. The comparison between a city's local centrality and its betweenness centrality reveal that whilst obvious betweenness centrality appears in cities like Lanzhou, Ningbo and Zhongshan (suggesting that they maintain relatively strong control over technological knowledge), in Shenzhen and Nanjing it is local centrality that stands out (showing that these cities have a prominent ability in technological cooperation). In comparing a city's local centrality and its closeness centrality, the closeness centrality value was always higher than the local centrality value; this reflects the weaker nature of a city's capability to carry out technological cooperation in comparison to its ability to avoid being controlled by other cities. By comparing closeness centrality and betweenness centrality, the study revealed that a given city's ability to avoid being controlled by other cities was always stronger than its ability to control technological knowledge, demonstrating that the ability of cities to control tech- nological knowledge is generally not strong enough in China (Fig. 3).

\subsection{Group structure based on cohesive subgroup analysis}

By using network cohesive subgroup analysis, a number of city groups were discovered to exist within the broader technological cooperation network. Within these city groups, whilst stronger technological connections existed between the cities within the group, connections between those cities and cities outside the group were weaker (Wasserman and Faust, 1994). The multi-weighted network was examined using Cluster Analysis with Ucinet software, and 5 cohesive subgroups (CSG) (Fig. 4) were classified.

According to the results: 1) the core subgroup CSG I encompassed a number of high-level cities, representing a national-level technological alliance made up of 15 cities. The top 8 cities with the highest local centrality value were Beijing, Shanghai, Shenzhen, Nanjing, Guangzhou, Suzhou, Tianjin and Zhongshan. Further, whilst they did not have high enough local centrality values, Luoyang and Harbin were also listed as part of the group due to their close connections with other
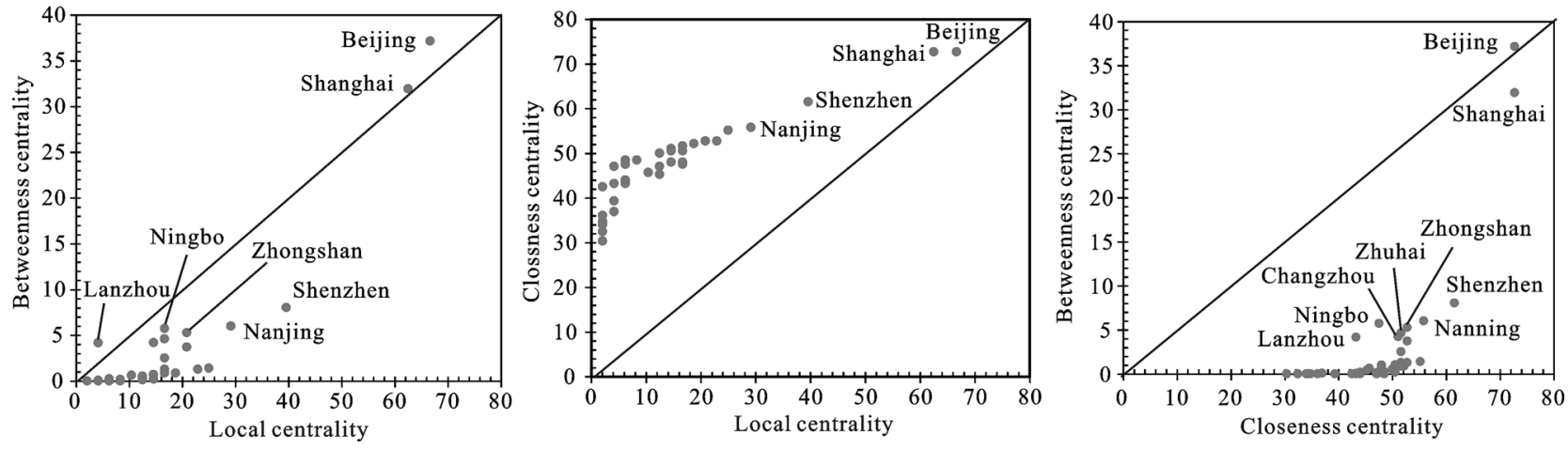

Fig. 3 Comparative analysis of three types of centrality in 49 cities 


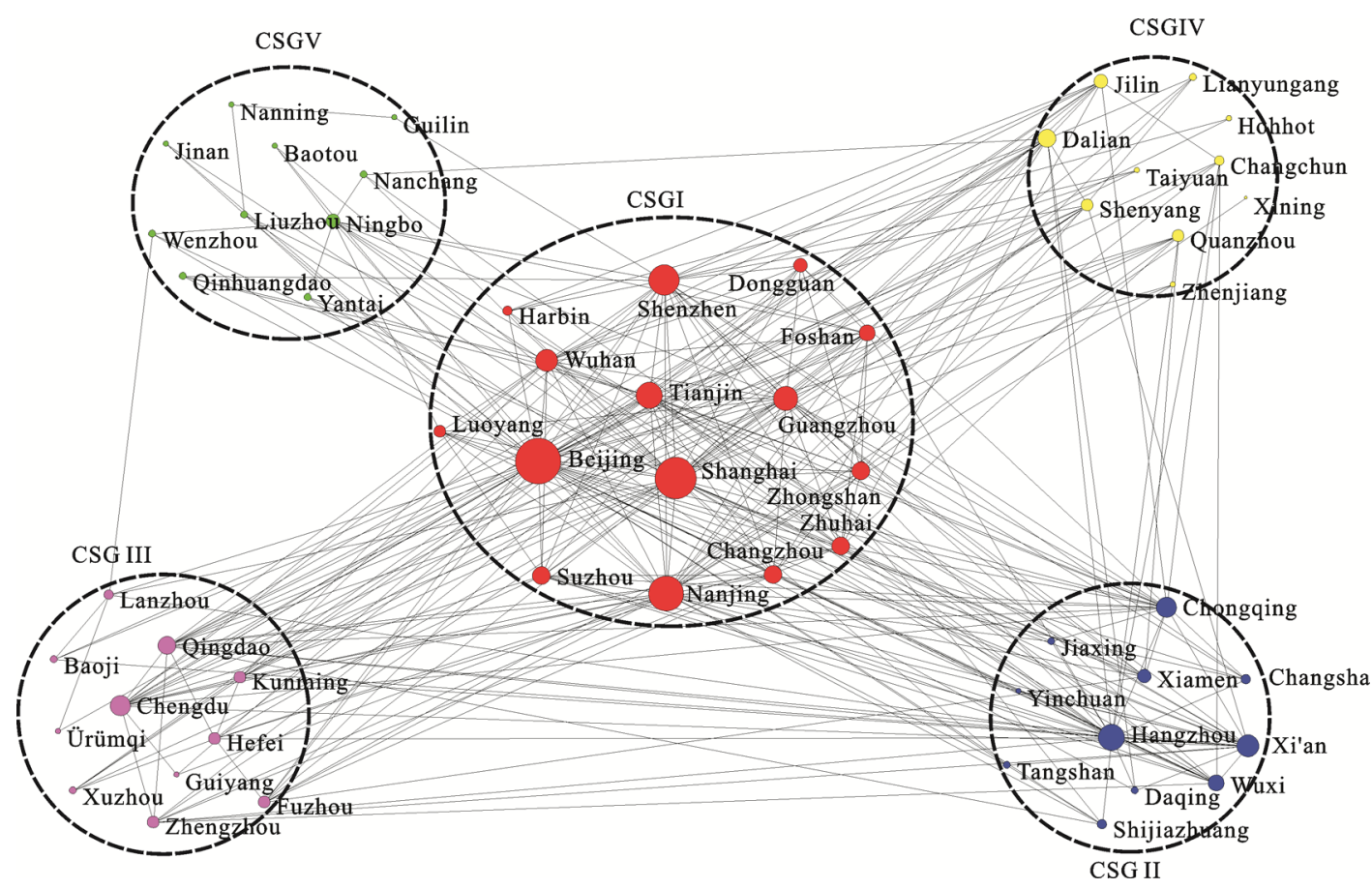

Fig. 4 Five cohesive subgroups (CSG) in Chinese city networks. Different subgroups are distinguished by different colors, and the point size suggests local centrality value. Haikou, Jingzhou and Lhasa are not shown here because they did not have patent cooperation with other cities

high-level cities in CSG I (these connections indicate a strong ability to establish technological cooperation alliances). 2) Although the other 4 CSGs maintained closer links to CSG I, they seldom had contact with each other. From the density matrix (Table 2), it can be seen that a number of differences exist in terms of the technological cooperation level within one group and between groups. Figure 4 shows a single link between CSG V and CSG III (that between Wenzhou and Lanzhou), and just one link between CSG V and CSG IV (that between Dalian and Nanchang). Despite the lack of evidence for current connections, in fact inter-group connections can bring more novel knowledge and be deeply beneficial to technological innovation. 3) The development of regional technological alliances is considered insufficient at present. On the one hand, cities that belonged to the core subgroup (CSG I) are scattered around the nation; on the other hand, within the other individual CSG, city communities in close technological relationship were very few. Considering the location factor, a number of technological alliances could be identified, namely, Hangzhou-Wuxi-Jiaxing within CSG II, Chengdu-Kunming within CSG III, Dalian-ShenyangJilin-Changchun within CSG IV, and Ningbo-YantaiWenzhou within CSG V.
Table 2 Density values within and between each cohesive subgroup

\begin{tabular}{crrrrr}
\hline & CSG I & CSG II & CSG III & CSG IV & CSG V \\
\hline CSG I & 32.41 & & & & \\
CSG II & 2.09 & 20.73 & & & \\
CSG III & 1.45 & 0.24 & 21.39 & & \\
CSG IV & 1.48 & 0.14 & 0.06 & 22.88 & \\
CSG V & 1.00 & 0.11 & 0.01 & 0.02 & 22.16 \\
\hline
\end{tabular}

\subsection{Network control structure based on struc- tural holes}

Structural Holes Analysis can reveal which cities have a greater capability to control the spread of key technological knowledge in city networks, and identify which cities and weak instances of cooperation would be more important to concentrate on in constructing a national technological cooperation network. Here, structural holes are defined as 'holes' that appear within the network structure (that is to say, within the city networks), whereby a certain city or cities carry out direct cooperation with a number of other cities, but do not cooperate directly with others. In such situation, this noncooperative association or disconnection phenomenon forms a 'hole' in the network. Sometimes within city networks the weak connections are more important than 
strong relations. Structural Holes Analysis is of help in identifying these important weak connections. Compared with other cities, cities located in structural holes have more competitive advantages, because structural holes can provide opportunities for information benefits and control benefits for cities located within them (Burt, 1992).

By calculating structural holes indicators for all the cities in the network (Table 3), we found 9 cities to be located in the periphery of the network. The value of all the structural holes indicators for these cities was 1 (Fig. 5). A number of conclusions can be drawn from a comparison of the structural holes indicators of other cities. 1) Cities within the eastern coastal region have stronger overall capability in terms of the control that they exert over the spread of technological knowledge. The top 3

Table 3 Comparison of structural holes indicators of 36 cities

\begin{tabular}{|c|c|c|c|c|c|c|c|}
\hline City & Effective size & Efficiency & Constraint & City & Effective size & Efficiency & Constraint \\
\hline Shanghai & 16.06 & 0.89 & 0.22 & Wuhan & 2.69 & 0.67 & 0.46 \\
\hline Beijing & 13.42 & 0.84 & 0.28 & Harbin & 2.21 & 0.74 & 0.60 \\
\hline Shenzhen & 11.31 & 0.87 & 0.25 & Hefei & 2.00 & 1.00 & 0.68 \\
\hline Zhongshan & 7.38 & 0.92 & 0.29 & Changchun & 2.00 & 1.00 & 0.57 \\
\hline Guangzhou & 6.10 & 0.76 & 0.35 & Chongqing & 2.00 & 1.00 & 0.52 \\
\hline Nanjing & 5.39 & 0.77 & 0.46 & Fuzhou & 1.78 & 0.89 & 0.59 \\
\hline Foshan & 5.01 & 0.83 & 0.37 & Yinchuan & 1.51 & 0.76 & 0.69 \\
\hline Suzhou & 4.29 & 0.72 & 0.42 & Baotou & 1.00 & 1.00 & 1.00 \\
\hline Zhuhai & 4.00 & 1.00 & 0.54 & Changsha & 1.00 & 1.00 & 1.00 \\
\hline Jilin & 4.00 & 1.00 & 0.30 & Dalian & 1.00 & 1.00 & 1.00 \\
\hline Tianjin & 3.98 & 0.66 & 0.48 & Lanzhou & 1.00 & 1.00 & 1.00 \\
\hline Dongguan & 3.92 & 0.78 & 0.44 & Lianyungang & 1.00 & 1.00 & 1.00 \\
\hline Qingdao & 3.89 & 0.97 & 0.42 & Liuzhou & 1.00 & 1.00 & 1.00 \\
\hline Wuxi & 3.72 & 0.75 & 0.42 & Luoyang & 1.00 & 1.00 & 1.00 \\
\hline Ningbo & 3.53 & 0.88 & 0.37 & Quanzhou & 1.00 & 1.00 & 1.00 \\
\hline Changzhou & 3.44 & 0.69 & 0.63 & Shenyang & 1.00 & 1.00 & 1.00 \\
\hline Xi'an & 3.06 & 0.76 & 0.45 & Xiamen & 1.00 & 1.00 & 1.00 \\
\hline Chengdu & 2.84 & 0.95 & 0.38 & Yantai & 1.00 & 1.00 & 1.00 \\
\hline
\end{tabular}

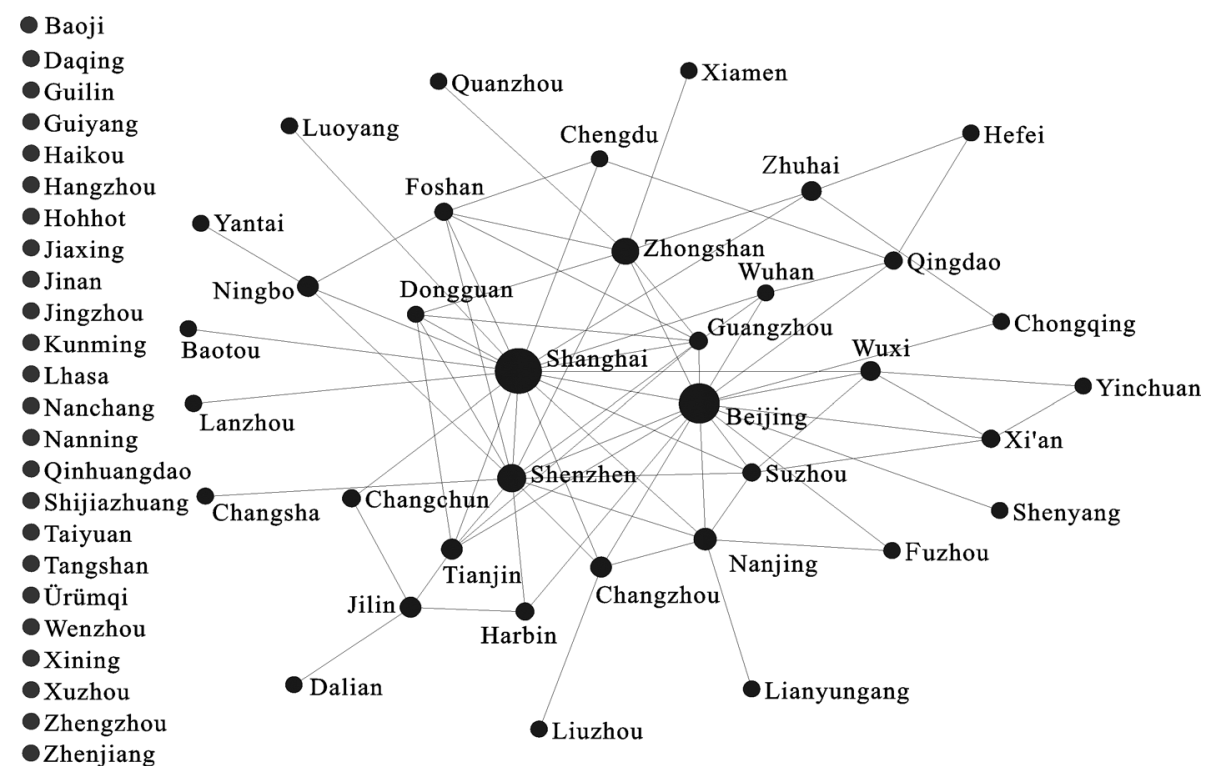

Fig. 5 Network structure with inter-city connectivity values of more than 10. Considering structural holes with high connection values to have more practical meanings, we excluded points with inter-city connectivity less than 10 in this study, which resulted in the identification of 24 isolated points, which in turn formed a network of 36 cities. The size of each point indicates the local centrality of that city 
cities were Shanghai, Beijing and Shenzhen, Further, overall capability appears to be more prominent in the cities of the Pearl River Delta region (Zhongshan, Guangzhou, Foshan and Zhuhai are included in top 10 list). 2) A number of cities showed significant dominating capabilities, specifically Shanghai, Beijing, Shenzhen and Zhongshan all demonstrated a strong ability and high level of control over the spread of key technological knowledge. Among these 4, Zhongshan showed a stronger controlling ability than Guangzhou. 3) The stronger the city's capability to control the spread of technological knowledge, the weaker the constraints on technological cooperation imposed by other cities. Moreover, cities able to exert the same level of control can also impose different levels of constraint. For instance, Chongqing, Changchun and Hefei were found to have the same effective size value, but the constraint level of Chongqing was found to be the lowest, with Changchun maintaining a medium level and Hefei the highest.

In order to identify important weak ties in the Chinese technological cooperation network, the relatively important cities (namely, Shanghai, Beijing, Shenzhen and Guangzhou) were excluded, which resulted in all connections to them being hidden. The remaining structure reflects a number of important weak connections (Fig. 6). For example, the cooperation between Dongguan and Tianjin acts as a bridge relating the southern China cities to the north-eastern cities, which is consid- ered particularly important; meanwhile, the cooperation between Nanjing and Suzhou links the two technological alliances together. Although cooperation between these cities is not strong, it plays a crucial role in building national technological cooperation network, which is worth significant further attention.

\subsection{Network dynamic structure based on prox- imity}

The dynamics of network connections constitute an important aspect of network evolution research. An understanding of such dynamics is beneficial both in terms of exploring the driving forces behind inter-city patent cooperation and in revealing the dynamics of technological cooperation network formation. Many forms of proximity can be used to explain the formation of innovation network, such as geographical proximity, cognitive proximity, organizational proximity, social proximity and institutional proximity. This diversity demonstrates the importance of forms of proximity beyond geography proximity to the development of interactive learning and innovation (Boschma and Frenken, 2010). This study combined Storper's (1997) tripartite 'regional innovation model' (and its basis of 'territory-technologyorganization') with an inter-city cooperation, putting forward three cooperation dynamics, namely, a geographical proximity dynamic, a technological proximity dynamic and a hierarchical proximity dynamic.

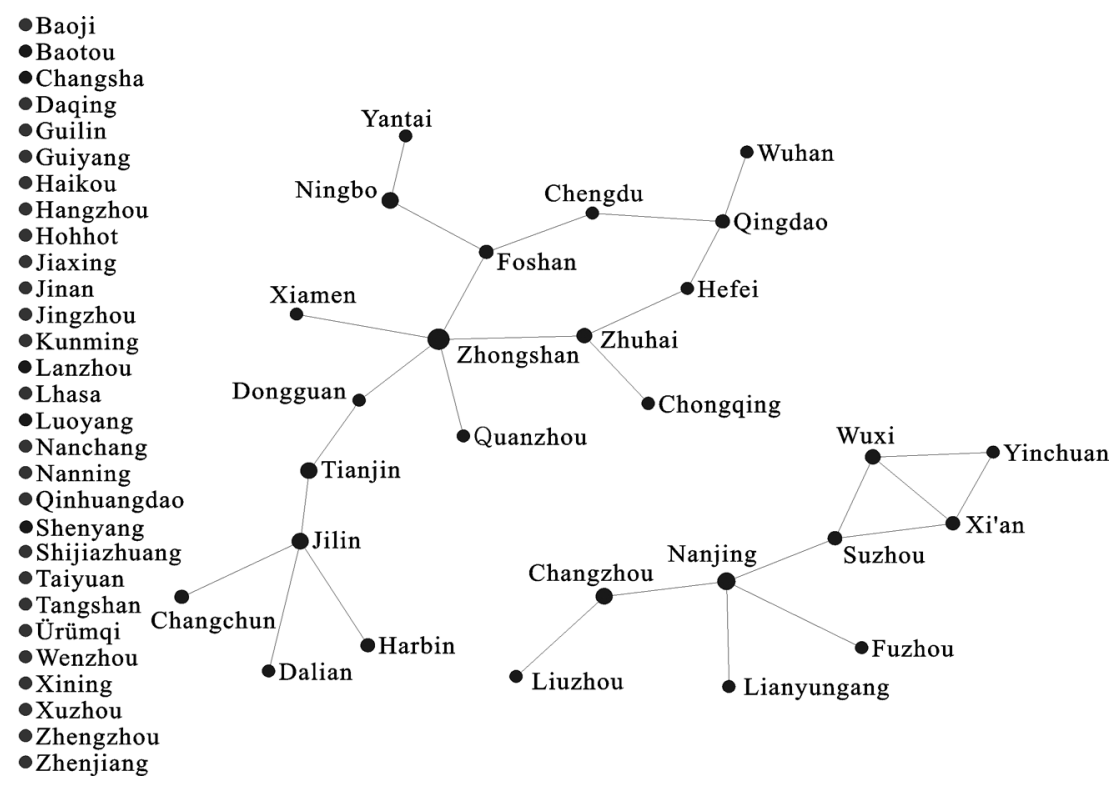

Fig. 6 Important weak ties in Chinese technological networks. It is the result after removing 4 relatively important cities (Shanghai, Beijing, Shenzhen and Guangzhou) in Fig. 5. Size of each point indicates the local centrality of that city 
First, the findings of this study reinforce the primary importance of the geographical proximity dynamic in urban technological cooperation (Ben Letaifa and Rabeau, 2013). By constructing both an inter-city distance matrix and an inter-city patent cooperation matrix, the amount of inter-city patent cooperation scales could be calculated at a range of different distances. The results of this calculation reveal that the closer the cities are, the larger the amount of cooperation is (Fig. 7). This suggests that the geographical proximity dynamic provides an important impetus for urban technological knowledge cooperation.

Second, most distant inter-city connections displayed hierarchical proximity. Three types of knowledge diffusion exist: expansion diffusion, hierarchical diffusion and relocation diffusion (Wallace et al., 1999). In that the same institutional foundations and comprehensive strengths often occur in cities with similar hierarchies, the hierarchical diffusion of technological knowledge is easier to facilitate than other forms of diffusion. This is the primary reason for promoting distant inter-city technological knowledge cooperation, as such, cooperation between most provincial capital cities is driven by hierarchical proximity.

Third, the essential reason for inter-city technological knowledge cooperation lies in the fact that technological proximity appears at various distance scales. Patent knowledge is highly professional technological knowledge; hence, such inter-city cooperation requires not only similar technological knowledge fundamentals, but also common demands for technological knowledge innovation (Boschma and Frenken, 2010). The cooperation evident between Luoyang and Wuxi, Jilin and Zhuhai falls into this category.

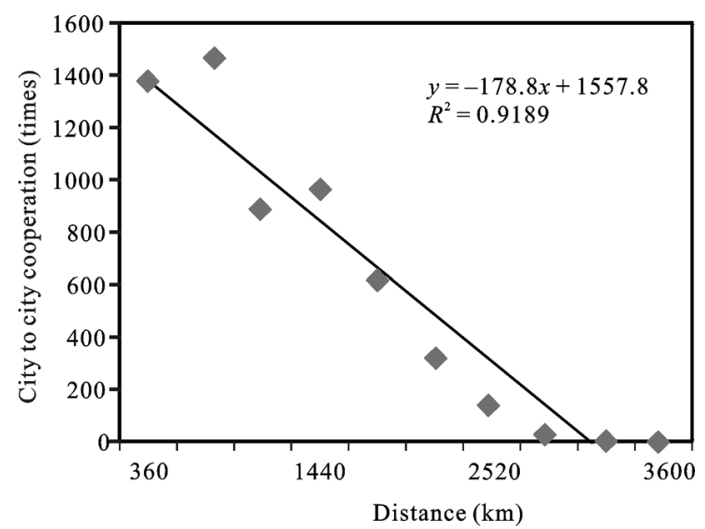

Fig. 7 Effect of geographical proximity on inter-city technological cooperation

\section{Conclusions and Discussion}

By exploring the structure of the Chinese city network, as it is driven by technological knowledge flow, the following results were produced. 1) Regional technological knowledge connections were found to occur mainly between Beijing, Wuhan, urban areas in Changjiang River Delta, urban areas in the Pearl River Delta and urban areas in Chengdu-Chongqing region. This resulted in a diamond network structure. However, as the central city in this structure, Wuhan does not perform very well as the broker and bridge. Meanwhile, north-western and north-eastern regions are lacking in hub cities. 2) Although invention patents were shown to represent the top-level form of technological knowledge in China, producing a knowledge cooperation network that constituted $43.69 \%$ of the entire network, the invention patent is still slightly inferior to the new utility model patent. This indicates a need for further enhancement. 3) In the Chinese technological cooperation network, Beijing and Shanghai form two absolute centers. However, regardless of their technological cooperation, their control of the spread of technological knowledge, and their approximation of high-tech centers, the stronger overall strength was shown to lie in the cities of the Pearl River Delta. 4) Closer connections were found to occur between important technology center cities, suggesting that whilst a national-level technological alliance has been formed, the development of regional technological alliances is insufficient. 5) Although some city pairs like Dongguan-Tianjin, Nanjing-Suzhou, Nanchang-Dalian and Lanzhou-Wenzhou have relatively weak connectivity, they play an important role in building the national technological cooperation network. It is easier to obtain both information benefits and controlling benefits through these links, which relate different regional alliances and technological alliances. 6) The three major dynamics of promoting inter-city technological cooperation are geographical proximity, technological proximity and hierarchical proximity.

According to these results and from the perspective of enhancing technological contacts, a number of suggestions for the improvement of China's ability to support and develop national and urban-level innovation are proposed here for governmental reference. First, inter-city connectivity and network density within the current development of technological network are not 
high, indicating that the overall level is actually relatively low. As such, the national government should actively encourage cross-city technological cooperation in order to promote the exchange of novel technological knowledge and to stimulate innovation. Secondly, judging from the spatial structure revealed by this study, the status of the north-western and north-eastern regions is not outstanding. In response, a number of appropriate cities should be selected to act as regional technology centers, in order to foster the development of a regional technological cooperation network. Last but not least, when cooperating actively with top-level technological cities (which in itself helps improve their ability to acquiring advanced technological knowledge), other cities should also occupy structural holes locations in order to improve their chances to control key technological knowledge. Such control in turn acts to enhance the capability of such cities to control the spread of technological knowledge, and would allow them to operate as bridges between different technological alliances.

In comparison to existing research on city networks, this study presents the following advances. Firstly, this study facilitates the structural analysis of city networks through its use of various, comprehensive network analysis methods such as spatial network analysis (Zhen et al., 2013), social network analysis (Burt, 1992) and network evolution analysis (Boschma and Frenken, 2010) in order to explore the various structures of city networks. Second, the research used new relational data to examine Chinese city networks. Existing Chinese city network research data mainly included virtual network data based on the gravity model (Leng et al., 2011), indirect network data gleaned from the GaWC group's analysis of service flow (Zhen et al., 2012) and traffic and telecommunication data based on statistical information (Wang et al., 2011). Departing from this tradition, this study used inter-city patent cooperation data from the Chinese Patent Database of the CNKI, engaging in a tentative exploration of inter-city direct connections. Third, this study applied knowledge about city networks to the practice of constructing an innovative country. Exploring the structure of city networks from a practical standpoint, through a focus on technological knowledge flows, this study and its findings can act as a reference for new practical activities and aid in the expansion of the research value of city networks. The next step in this line of research is to track changes in this structure, analyze network evolution based on historical data, and to compare the technological knowledge flows network with other flow networks.

\section{References}

Barber M J, Scherngell T, 2013. Is the European R\&D network homogeneous? Distinguishing relevant network communities using graph theoretic and spatial interaction modelling approaches. Regional Studies, 47(8): 1283-1298. doi: 10.1080/ 00343404.2011.622745.

Ben Letaifa S, Rabeau Y, 2013. Too close to collaborate? How geographic proximity could impede entrepreneurship and innovation. Journal of Business Research, 66(10): 2071-2078. doi:10.1016/j.jbusres.2013.02.033.

Boschma R A, Frenken K, 2010. The spatial evolution of innovation networks: a proximity perspective. In: Boschma $\mathrm{R}$ A et al. (eds.). Handbook of Evolutionary Economic Geography. Cheltenham: Edward Elgar, 120-135.

Bourne L S, 1975. Urban Systems: Strategies for Regulation. Oxford: Oxford University Press, 26-50.

Burt R S, 1992. Structural Holes: the Social Structure of Competition. Cambridge, Mass: Harvard Business School Press, 3-40.

Camagni R P, Capello R, 2004. The city network paradigm: Theory and empirical evidence. In: Capello $\mathrm{R}$ et al. (eds.). Urban Dynamics and Growth. Netherlands: Elsevier B.V., $1-10$.

Castells M, 1989. The Information City. Oxford: Basil Blackwell, 3-8.

Choi J H, Barnett G A, Chon B S, 2006. Comparing world city networks: A network analysis of Internet backbone and air transport intercity linkages. Global Networks-a Journal of Transnational Affairs, 6(1): 81-99. doi: 10.1111/j.1471-0374. 2006.00134.x.

Dai Teqi, Jin Fengjun, 2008. Spatial interaction and network structure evolvement of cities in terms of China's rail passenger flows. Chinese Geographical Science, 18(3): 206-213. doi: 10.1007/s11769-008-0206-2.

Derudder B,Witlox F, 2005. An appraisal of the use of airline data in assessing the world city network: a research note on data. Urban Studies, 42(13): 2371-2388. doi: 10.1080/004209805 00379503.

Eslami H, Ebadi A, Schiffauerova A, 2013. Effect of collaboration network structure on knowledge creation and technological performance: the case of biotechnology in Canada. Scientometrics, 97(1): 99-119. doi: 10.1007/s11192013-1069-6.

Esparza A X, Krmenec A J, 2000. Large city interaction in the US urban system. Urban Studies, 37(4): 691-709. doi: 10.1080/00420980050003973.

Fischer M M, Scherngell T, Jansenberger E, 2006. The geography of knowledge spillovers between high-technology firms in 
Europe: Evidence from a spatial interaction modeling perspective. Geographical Analysis, 38(3): 288-309. doi: 10. 1111/j.1538-4632.2006.00687.x

Hou H, Kretschmer H, Liu Z, 2008. The structure of scientific collaboration networks in Scientometrics. Scientometrics, 75(2): 189-202. doi: 10.1007/s11192-007-1771-3.

Jacobs W, Ducruet C, De Langen P, 2010. Integrating world cities into production networks: the case of port cities. Global Networks-a Journal of Transnational Affairs, 10(1): 92-113. doi: 10.1111/j.1471-0374.2010.00276.x

Lei X P, Zhao Z Y, Zhang X et al., 2013. Technological collaboration patterns in solar cell industry based on patent inventors and assignees analysis. Scientometrics, 96(2): 427-441. doi: 10.1007/s11192-012-0944-x

Leng Bingrong, Yang Yongchun, Li Yingjie et al., 2011. Spatial characteristics and complex analysis: a perspective from basic activities of urban networks in China. Acta Geographica Sinica, 2(66): 199-211. (in Chinese).

Leydesdorff L, Persson O, 2010. Mapping the Geography of science: distribution patterns and networks of relations among cities and institutes. Journal of the American Society for Information Science and Technology, 61(8): 1622-1634. doi: 10.1002/Asi.21347

Liefner I, Hennemann S, 2011. Structural holes and new dimensions of distance: The spatial configuration of the scientific knowledge network of China's optical technology sector. Environment and Planning A, 43(4): 810-829.

Lu Luchang, Huang Ru, 2012. Urban hierarchy of innovation capability and inter-city linkages of knowledge in post-reform China. Chinese Geographical Science, 22(5): 602-616. doi: 10.1007/s11769-012-0555-8

Matthiessen C W, Schwarz A W, Find S 2002. The ups and downs of global research centers. Science, 297(5586): 1476-1477. doi: 10.1097/CCM.0b013e3181d16b00.

Matthiessen C W, Schwarz A W, Find S, 2010. World cities of scientific knowledge: systems, networks and potential dynamics. An analysis based on bibliometric indicators. Urban Studies, 47(9): 1879-1897. doi: 10.1177/0042098010372683.

Mo Huihui, Wang Jiaoe, Jin Fengjun, 2009. Identifying centrality in the air transportation network of China. Transportation and Geography, 2: 493-502. doi: 10.1371/journal.pone.0066732.

Nascimbeni F, 2013. Collaborative knowledge creation in development networks: lessons learnt from a transnational. The Journal of Community Informatics, 9(3): 1-8.
Neal Z, 2010. Refining the Air Traffic Approach to City Networks. Urban Studies, 47(10): 2195-2215. doi: 10.1177/ 0042098009357352

Storper M, 1997. The Regional World: territorial Development in a Global Economy. New York: Guilford press. 3-25.

Tang L, Hu G Y, 2013. Tracing the footprint of knowledge spillover: Evidence from U.S.: China collaboration in Nanotechnology. Journal of the American Society for Information Science and Technology, 64(9): 1791-1801. doi: 10.1002/Asi.22873

Taylor P J, 2001. Specification of the world city network. Geographical Analysis, 33(2): 181-194.

Taylor P J, Catalano G, Walker D R F, 2002. Measurement of the world city network. Urban Studies, 39(13): 2367-2376.

Thompson P, Fox-Kean M, 2005. Patent citations and the geography of knowledge spillovers: a reassessment. American Economic Review, 95(1): 450-460. doi: 10.1257/00028280 53828509.

Vinciguerra S, Frenken K,Valente M, 2010. The Geography of internet infrastructure: an evolutionary simulation approach based on preferential attachment. Urban Studies, 47(9): 1969-1984.

Wallace R, Wallace D, Ullmann J E et al., 1999. Deindustrialization, inner-city decay, and the hierarchical diffusion of AIDS in the USA: how neoliberal and cold war policies magnified the ecological niche for emerging infections and created a national security crisis. Environment and Planning A, 31(1): 113-139. doi: 10.1068/A310113

Wang J E, Mo H H, Wang F H et al., 2011. Exploring the network structure and nodal centrality of China's air transport network: A complex network approach. Journal of Transport Geography, 19(4): 712-721. doi: 10.1016/j.jtrangeo.2010. 08.012.

Wasserman S, Faust K, 1994. Social Network Analysis: Methods and Applications. New York and Cambridge, ENG: Cambridge University Press.

Zhen Feng, Wang Bo, Chen Yingxue, 2012. China's city network characteristics based on social network space: an empirical analysis of Sina Micro-blog. Acta Geographica Sinica, 67(8): 1031-1043. (in Chinese).

Zhen Feng, Wang Xia, Yin Jun et al., 2013. An empirical study on Chinese city network pattern based on producer services. Chinese Geographical Science, 23(3): 274-285. doi: 10.1007/ s11769-013-0595-8. 